\title{
Giant Hall Effect in Nonmagnetic Granular Metal Films
}

\author{
X. X. Zhang, * Chuncheng Wan, H. Liu, Z. Q. Li, and Ping Sheng \\ Department of Physics and Institute of Nano Science \& Technology, The Hong Kong University of Science and Technology, \\ Clear Water Bay, Kowloon, Hong Kong, China \\ J. J. Lin \\ Institute of Physics, National Chiao Tung University, Hsinchu 300, Taiwan
}

(Received 26 April 2000)

\begin{abstract}
Nearly 3 orders of magnitude enhancement in the Hall coefficient is observed in $\mathrm{Cu}_{x}-\left(\mathrm{SiO}_{2}\right)_{1-x}$ granular films. This large enhancement of the Hall coefficient not only is significantly larger than the prediction of the classical percolation theory, but also occurs at a metal concentration identified to be the quantum percolation threshold. Measurements of the electron dephasing length and magnetoresistance, plus the TEM characterization of microstructures, yield a physical picture consistent with the mechanism of the local quantum interference effect.
\end{abstract}

DOI: $10.1103 /$ PhysRevLett.86.5562

As a basic material constant, the Hall coefficient is generally indicative of the density and sign of the charge carriers. Thus, in granular metals, as the metal concentration decreases, the lower carrier density is expected to yield an enhanced Hall coefficient which peaks at the percolations threshold with a factor of $\sim 30$ for $\sim 1 \mu \mathrm{m}$ thick films $[1,2]$. Recently, however, it was found that in the magnetic $(\mathrm{NiFe})-\mathrm{SiO}_{2}$, and $\mathrm{Fe}-\mathrm{SiO}_{2}$ granular films [2-5], the extraordinary Hall coefficient was enhanced by a factor of $10^{4}$ when the metal volume fraction is close to $x=0.53$ (the classical percolation threshold). An especially intriguing feature of this discovery is that, even after magnetic saturation, the ordinary Hall coefficient was still observed to increase by almost 3 orders of magnitude [4], suggesting a magnetic-independent mechanism could be operative.

In this Letter, we focus on the origin of the ordinary giant Hall effect (GHE) by studying the nonmagnetic $\mathrm{Cu}-\mathrm{SiO}_{2}$ granular system. We find the same 3 orders of magnitude enhancement in the Hall coefficient. By carrying out measurements on the electron dephasing length and the magnetoresistance (MR), and by characterizing our samples by transmission electron microscope (TEM) pictures, we find the Hall coefficient to peak at the quantum percolation threshold. Based on the picture that inhomogeneities (due to the small substructures) inside a dephasing length would necessarily cause local quantum interference and thereby modify the effective local properties, we show that all experimental data can be quantitatively accounted for within this simple framework. In particular, when the small substructures are suppressed through annealing, the GHE is shown to disappear, in agreement with the theoretical prediction [6].

$\mathrm{Cu}-\mathrm{SiO}_{2}$ granular films with different metal volume fractions were fabricated by using the cosputtering technique with a glass or Kapton substrate, at a temperature of $50{ }^{\circ} \mathrm{C}$. The base pressure of the chamber was kept below $2 \times 10^{-7}$ Torr. The films deposited on the glass were used for transport measurements and the films on Kapton
PACS numbers: 72.20.My, 71.30. $+\mathrm{h}, 72.80 . \mathrm{Tm}, 73.50 . \mathrm{Jt}$

were for composition determination. The metal volume fraction $x$ for all the films was obtained from energydispersive $\mathrm{x}$-ray spectroscopy analysis. The dc resistance was measured by using the standard four-probe technique, and the Hall resistance was measured by using the five-contacts method.

More than 25 samples, with $0.4<x<0.81$ and a thickness of $\sim 1 \mu \mathrm{m}$, have been fabricated and characterized. Longitudinal resistivity, $\rho_{x x}$, was measured as a function of temperature between 4 and $350 \mathrm{~K}$. As quantum effects, such as hopping conduction or electron localization, are more dominant at low temperatures, we identify the (classical) percolation threshold by focusing on the temperature dependence of room temperature $(300 \mathrm{~K})$ resistivity [7]. We find the room temperature $d \ln \rho / d \ln T$ to change sign at $x=x_{c}=0.43$, indicative of the percolation threshold. This is corroborated by the fact that, at $x=0.42, \rho_{x x}$ exhibits the $\ln \sigma \propto-1 / \sqrt{T}$ hopping conduction behavior [8] and is 2 orders of magnitude larger than that for $x=0.45$. While not shown, we have also measured the magnetoresistance. The negative $\mathrm{MR}=[\rho(H)-\rho(0)] / \rho(0)$, observed at $5 \mathrm{~K}$ for various compositions, is indicative of the electron weak localization effect. In particular, the MR magnitude is noted to peak above the percolation threshold.

The Hall resistivity, $\rho_{x y}$, for all samples was measured in a magnetic field $-4.6 \leq H \leq 4.6 \mathrm{~T}$ and at temperatures ranging from 2.5 to $50 \mathrm{~K}$. A perfect linear dependence of $\rho_{x y}$ on the magnetic field was observed. In Fig. 1, the measured $\rho_{x y}$ at $5 \mathrm{~K}$ is plotted versus $x-x_{c}$. It is seen that $\rho_{x y}$ increases monotonically with decreasing $x$, reaching a maximum of $R_{0}=1.84 \times 10^{-8} \mathrm{~m}^{3} / \mathrm{C}$ at $x \sim 0.513$, and plateaus after that. The ratio of the highest value to the lowest is 700, almost 3 orders of magnitude, and this enhancement is nearly independent of the temperature up to $50 \mathrm{~K}$. In magnetic granular films, the ordinary Hall coefficient is noted to increase by about 640 times [4], similar to what we find here. The maximum 


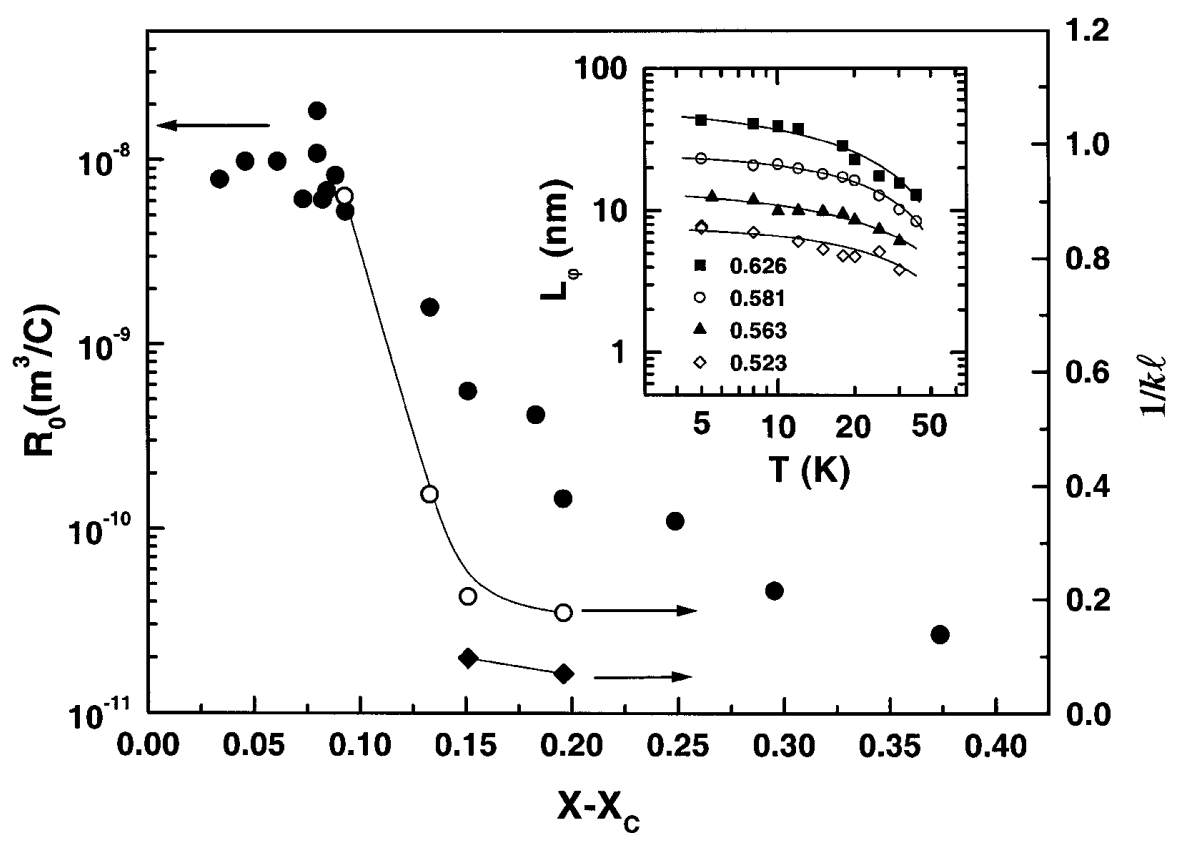

FIG. 1. The Hall resistivity plotted as a function of $x-x_{c}$, on a log-linear scale. The values of $1 / k \ell$ for several typical samples are also shown; open circles are for the lower bound of $\ell=1 \mathrm{~nm}$, solid diamonds are for the upper bound of $\ell=10 \mathrm{~nm}$. The inset shows the temperature dependence of the dephasing length for several samples.

$R_{0}=1.84 \times 10^{-8} \mathrm{~m}^{3} / \mathrm{C}$ in our sample is larger than that observed in Al-Ge [9], $\mathrm{Au}-\mathrm{SiO}_{2}, \mathrm{~W}-\mathrm{SiO}_{2}$ [10], and $\mathrm{Al}$ granular films [11], where the enhancement is about 100 or less. However, we should note that there are large fluctuations in the measured maximum $R_{0}$. Thus, a careful search in other granular systems might yield enhancement factors similar to what we have observed.

The measured enhancement in Hall coefficient is much larger than that expected by the classical percolation theory, where the maximum predicted enhancement factor (occurring at the percolation threshold) is on the order of $(T / a)^{(g / \nu)}$, where $T$ is the thickness of the film, and $a$ is the size of granules. Here, $g$ is the critical exponent of 3D Hall resistivity, and $\nu$ is the exponent for the 3D correlation length. The ratio $g / \nu$ is approximately given by $0.4 / 0.9 \sim 0.45$ [1]. By taking $T=1 \mu \mathrm{m}$, the thickness of our films, and a minimum value of $a=1 \mathrm{~nm}$, we get $(T / a)^{(g / \nu)} \sim 30[2]$.

Besides the magnitude of the enhancement factor, another intriguing feature is that the maximum Hall coefficient does not occur at the percolation threshold, $x_{c}=$ 0.43 , but rather at a higher value of $x=0.51$, unexpected within the classical picture. Below, we show that MR data, when coupled with electron dephasing length measurements and TEM characterization of the microstructures, identify $x \sim 0.51$ to be close to the quantum percolation threshold.

Electron dephasing length of our samples was obtained by the standard approach of measuring the magnetoresistance in low magnetic fields $(<0.5 \mathrm{~T})$, over a temperature range between $5-35 \mathrm{~K}$, and then least-squares fitting the data to the 3D weak-localization predictions [12-15] for extracting the electron dephasing length $L_{\varphi}(T)$. The results are shown in the inset of Fig. 1. It is seen that $L_{\varphi}$ increases with decreasing temperature and increasing metal volume fraction. It varies from $\sim 10 \mathrm{~nm}$ up to slightly below $100 \mathrm{~nm}$. We have also characterized the microstructure of our samples by means of TEM pictures. In Fig. 2(a), it is seen that, for the sample with $x=0.513$ and a corresponding large Hall coefficient, there are many small substructures on the order of $1 \mathrm{~nm}$. Since this is considerably smaller than the measured $L_{\varphi} \approx 8 \mathrm{~nm}$, it follows that, within a footprint on the order of $L_{\varphi} \times L_{\varphi} \times L_{\varphi}$ [seen as a dashed circle on Fig. 2(a)], the local quantum interference must necessarily be present. In Fig. 2(b), the high resolution TEM picture of the same sample, annealed at $450{ }^{\circ} \mathrm{C}$ for $1 \mathrm{~h}$ is seen to have a grain size $\sim 10 \mathrm{~nm}$, with a clear suppression of the small substructures. The Hall coefficient of the annealed sample was measured to be more than 2 orders of magnitude smaller than before; i.e., the GHE has disappeared. There is thus a clear-cut correlation between the presence of small substructures, dephasing length, and the GHE.

An important indicator of electron scattering/ localization effect is the product $k \ell$, where $k$ is the electron wave vector (renormalized by multiple scattering effects) at the Fermi level, and $\ell$ is its scattering mean-free path. From the measured MR and the dephasing length, and by setting the scattering mean-free path to be the size of the small substructures $(1 \mathrm{~nm}$ at $x=0.513)$, we can directly deduce the value of $k \ell$ [16]. This is shown by the open circles in Fig. 1. It is seen that the composition where the Hall coefficient peaks corresponds with $k \ell \sim 1$, i.e., the quantum percolation threshold (or the mobility edge) [16]. For high metal concentrations, the $k \ell$ increases considerably even if we still assume the 


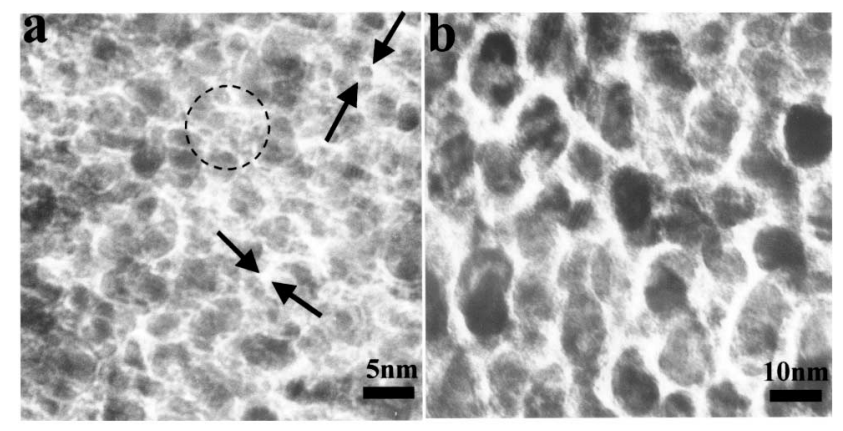

FIG. 2. High resolution TEM pictures of the sample with $x=$ 0.513. (a) The image for the as-prepared sample, in which small substructures of insulating particles and metallic particles, on the scale of 1 to $3 \mathrm{~nm}$, are clearly seen (as indicated by the arrows). The dashed circle indicates schematically the size of a footprint on the order of one dephasing length. Inhomogeneities inside the dephasing length are clearly seen. (b) The image of the same sample after annealing at $450{ }^{\circ} \mathrm{C}$ for $1 \mathrm{~h}$. The feature size is greatly increased (to about $10 \mathrm{~nm}$ ), with a narrower size distribution and a clear suppression of small substructures.

mean-free path to be $1 \mathrm{~nm}$. By assuming $\ell$ to have the upper bound value of $10 \mathrm{~nm}$ would make $k \ell$ even larger (indicated by solid diamonds). We have also measured the MR of the annealed sample shown in Fig. 2(b). The resulting $k \ell$, by using a mean-free path of $10 \mathrm{~nm}$, is $\sim 13$, i.e., the scattering/interference effect is greatly reduced.

Our experimental results consistently indicate a strong correlation between the GHE and the electron wave scattering/interference effect. In particular, if $L_{\varphi}$ is less than the feature/grain size, such as in the annealed samples, then dephasing would occur inside each grain. Electrical conduction between the connected metallic grains would then be classical in character, i.e., there should be no GHE. This is in contrast to the reverse case, where the interference effect would be present locally. Based on this picture, we have carried out theoretical calculations by using the following physical model. Because of the wave nature of the electrons, the Hall coefficient results from a combination of the classical Hall effect as well as the local interference of the electronic wave functions. Thus, the effective local Hall conductivity $\sigma_{H}=\sigma_{x y}=-\sigma_{y x}$, as well as the (longitudinal) conductivity $\sigma=\sigma_{x x}=\sigma_{y y}=\sigma_{z z}$, can be calculated by using the quantum percolation model [17] on finite-sized samples. Details of the calculations are given in [6]. As the interference effect is insensitive to the sign of the charge carrier but sensitive to the detail of the local microstructure, it follows that the calculated $\sigma_{H}$ can be either positive or negative when considered over all possible random, local microstructures. Two calculated distributions of the Hall conductances are shown in the inset of Fig. 3, where we have considered a $10 \times 10 \times 10$ finite-size quantum percolation model (i.e., substructure sizes about 10 times smaller than the dephasing length) [18]. At concentrations far above the percolation threshold, the distribution is broad and peaks at a negative value. For concentrations close to the quantum percolation threshold but above the (classical geometric) percolation threshold, the distribution is very symmetrical and peaks approximately at zero. While not shown, distributions of local (longitudinal) conductivities can also be similarly obtained [6]. The knowledge of the local (Hall and longitudinal) conductivity distributions at every concentration enables us to calculate the macroscopic conductivity tensor, where we use the classical (Kirchhoff) conditions of $\nabla \times \vec{E}=0$ and $\nabla \cdot \vec{j}=0$, where $\vec{j}=\tilde{\sigma} \vec{E}$. Here, $\tilde{\sigma}$ is the local conductivity tensor (with components $\sigma_{H}=\sigma_{x y}=-\sigma_{y x}$ and $\sigma=\sigma_{x x}=\sigma_{y y}=\sigma_{z z}$ ). By combining the two conditions to yield $\nabla \cdot \tilde{\sigma} \nabla \phi=0$, where $\phi$ denotes the electrical potential, and discretizing the model, we have numerically solved the problem by allocating on each discretized node a local $\tilde{\sigma}$ in accordance with the prior-calculated distributions. The solution of the classical network problem yields the macroscopic Hall resistivity $\bar{\rho}_{H}=1 / \bar{\sigma}_{H}$ and the macroscopic resistivity $\bar{\rho}=1 / \bar{\sigma}$. To compare the predictions of the theory with the experiment, we eliminate the concentration $x$ by cross-plotting the Hall coefficient as a function of $\bar{\rho}$ in Fig. 3. Excellent quantitative agreement is seen. The GHE can therefore be heuristically understood as follows. Since $R_{0} \sim 1 / \bar{\sigma}_{H} \sim 1 /\langle n\rangle e c$, a symmetric distribution in local Hall conductivities effectively implies an overall decrease in the averaged charge carrier density and, hence, an increase in the Hall coefficient, because $R_{0} \sim 1 /\langle n\rangle e c$, where $\langle n\rangle \sim 0$ denotes the average charge carrier density. This also gives a plausible explanation to the temperature independence of the observed GHE up to $50 \mathrm{~K}$, because the temperature variation is associated with the magnitude of the dephasing length. While the distribution of the local Hall conductivity can vary with the size of coherence region as defined by the dephasing length, the mean of the distribution $(\sim\langle n\rangle)$, which depends on the degree of asymmetry in the distribution, may remain relatively robust up to a point. Moreover, the interference component increases in importance as $x$ approaches the quantum percolation threshold, which is the point at which the interference-induced wave function localization occurs (at the Fermi level). Below the quantum percolation threshold, interference has the effect of changing the localization length. As $L_{\varphi}$ is only a few feature sizes in our case, the localization length variation is not expected to be significantly perceivable on that scale, and a plateau in Hall resistivity results. As the quantum percolation threshold is above the classical percolation threshold, it follows that the GHE does not "see" the classical $x_{c}$, but rather peaks at or close to the quantum percolation threshold. Local interference is also expected to affect the resistivity by modifying the two-component composite picture to one that has a distribution of local conductivities. Moreover, this distribution is expected to have an increased number of small conductivities due to the localizing nature of the interference effect. Nonuniversal critical behavior may therefore result.

This work is supported by Hong Kong RGC Grant No. HKUST6159/99P. The authors thank Dr. T. K. Ng 


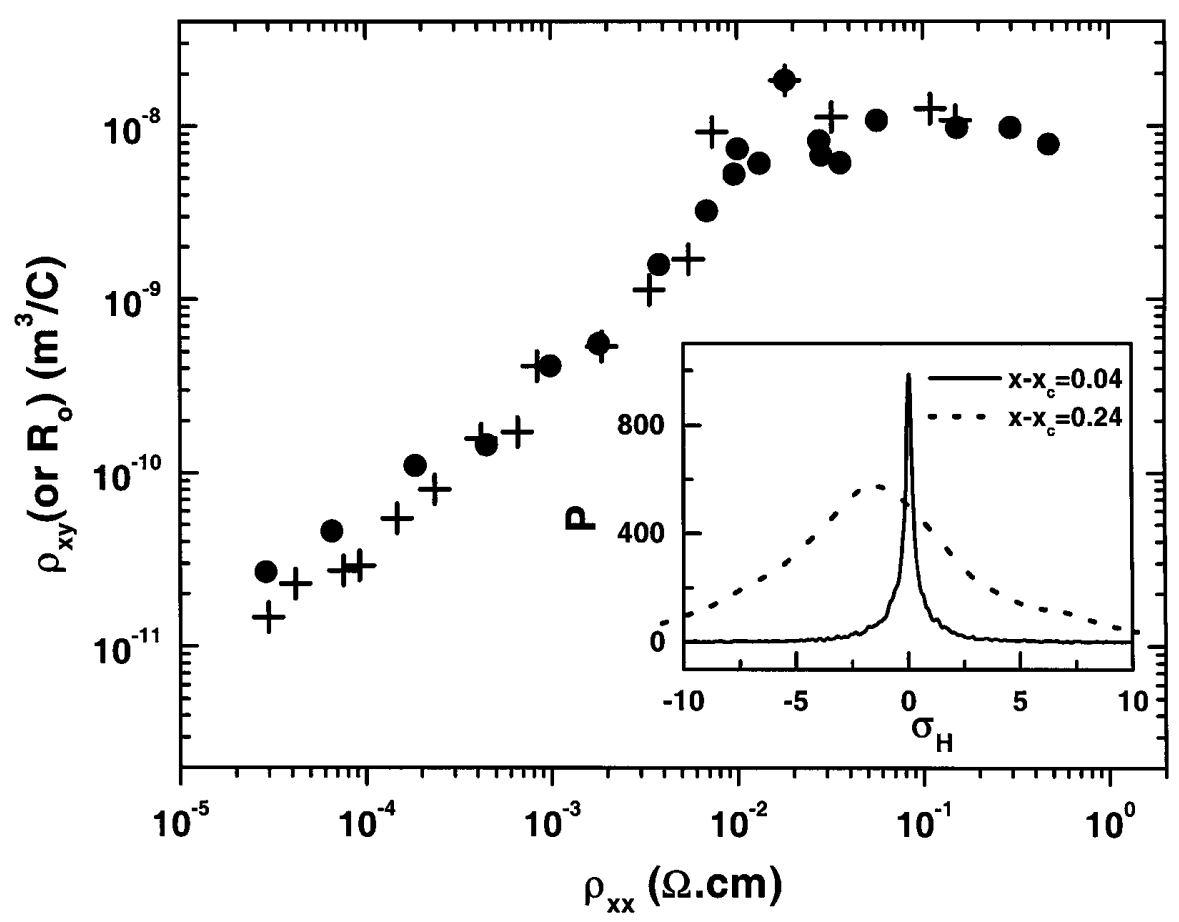

FIG. 3. The Hall coefficient cross-plotted as a function of $\rho_{x x}$. Experimental data are shown by solid circles and the theory points are indicated by crosses. The theory and experimental data are normalized at one point - the peak value of the Hall coefficient and its associated (longitudinal) resistivity. Excellent quantitative agreement is seen. Inset: Distributions of local Hall conductivities at two metal concentrations, calculated from the $10 \times 10 \times 10$ quantum percolation model through the Kubo formula. The distribution is noted to be nearly symmetrical for the concentration close to the percolation threshold. Here $x_{c} \cong 0.31$ is the theoretical model.

and Dr. Alec Pakhomov for useful discussions. The help offered by Dr. K. K. Fung and Dr. H.P. Xu on TEM, by Dr. G. H. Wen on the annealing experiment, and by Dr. A. K. Meikap and Mr. T. J. Li on the dephasing length experiment is hereby gratefully acknowledged.

*Author to whom correspondence should be addressed. Email address: phxxz@ust.hk

[1] D. J. Bergman and D. Stroud, Solid State Phys. 46, 149 (1992); D. Stauffer and A. Aharony, Introduction to Percolation Theory (Taylor \& Francis, Bristol, 1994).

[2] X. N. Jing et al., Phys. Rev. B 53, 14032 (1996).

[3] A. B. Pakhomov, X. Yan, and B. Zhao, Appl. Phys. Lett. 67, 3497 (1995).

[4] A. B. Pakhomov and X. Yan, Solid State Commun. 99, 139 (1996); Physica (Amsterdam) 229A, 402 (1996); A. B. Pakhomov et al., 241, 344 (1997).

[5] B. A. Aronzon et al., Phys. Status Solidi B 205, 151 (1998); B. A. Aronzon et al., JETP Lett. 70, 90 (1999).

[6] C. Wan and P. Sheng (to be published).

[7] It is common in granular systems to see insulating temperature variation at low temperatures and metallic behavior at high temperatures, for metal concentrations that fall between the quantum and classical percolation thresholds. This is due to an increased local quantum interference effect at low temperatures, associated with the increased dephasing length. Thus, at $T=0 \mathrm{~K}$, the conductivity threshold should be at the quantum percolation threshold, whereas, at $T=\infty$, the conductivity threshold should be at the classical, or geometric (connectivity) threshold.
[8] X. X. Zhang, H. Liu, and A. B. Pakhomov, Physica (Amsterdam) 279B, 81-83 (2000).

[9] U. Dai, A. Palevski, and G. Deutscher, Phys. Rev. B 36, 790 (1987).

[10] E. K. Sichel and J. I. Gittleman, Solid State Commun. 42, 75 (1982).

[11] B. Bandyopadhyay et al., Phys. Rev. B 26, 3476 (1982).

[12] See Eq. (3) in Fukuyama and Hoshino, [J. Phys. Soc. Jpn. 50, 2131 (1981)], and Eq. (1) in Wu and Lin, [Phys. Rev. B 50, 385 (1994)]. Since our film thickness is much larger than the dephasing length, the weak localization effects are $3 \mathrm{D}$ in character, and the measurement is thus independent of the applied field orientation.

[13] K. C. Mui, P. Lindenfeld, and W. L. McLean, Phys. Rev. B 30, 2951 (1984).

[14] A. G. Aronov, M.E. Gershenzon, and Yu.E. Zhuravlev, Sov. Phys. JETP 60, 554 (1984).

[15] Y. L. Zhong and J. J. Lin, Phys. Rev. Lett. 80, 588 (1998).

[16] P. Sheng, Introduction to Wave Scattering, Localization, and Mesoscopic Phenomena (Academic, Boston, 1995), p. 251.

[17] S. Kirkpatrick, Phys. Rev. B 6, 3598 (1972); C. M. Soukoulis, E. N. Economou, and G. S. Grest, Phys. Rev. B 36, 8649 (1987). The Hall conductivity in the quantum percolation model is calculated via the Kubo formula, by numerically diagonalizing the Hamiltonian of the finite-sized sample, and carrying out the summation over all excited states.

[18] If the grain size is large (such as in the annealed sample), then the distribution would be bimodal in character (either metal or insulator), and classical behavior prevails. 